\title{
THE INTERACTION OF A MAGNETOELASTIC SHEAR WAVE WITH LONGITUDINAL CAVITIES IN A CONDUCTING LAYER
}

\author{
V. I. Ostrik and L. A. Fil'shtinskii
}

UDC 539.3

\begin{abstract}
We study the influence of a strong magnetic field on the interaction of a shear wave with longitudinal cylindrical cavities in an elastic ideally conducting layer. The resulting singular integral equation of the boundary-value problem under consideration is implemented numerically for the case of a single cavity. We present the results of computation of the stresses on the edge of a circular cavity and an elliptical cavity.
\end{abstract}

In connection with the application of superconducting matter in engineering it is necessary to take account of the influence of electromagnetic fields on the dynamic stress of structural elements. This influence is especially noticeable when the bodies contain stress concentrators of crack, hole, and inclusion type.

Certain problems in the diffraction of magnetoelastic waves at straight-line cracks in dia(para)magnetic matter were studied in [7-9]. In the present paper we study the problem of diffraction of a magnetoelastic wave at holes. The corresponding boundary-value problem of magnetoelasticity reduces to a singular integral equation that is realized numerically by the method of mechanical quadratures. We give numerical results that characterize the influence of the magnitude of the preliminary magnetic field, the shape of the hole, and the frequency of the excitation on the stress concentration.

We consider an ideally conducting elastic layer $(0 \leq x \leq a,-\infty<y<\infty,-\infty<z<\infty)$ in a static magnetic field of intensity $\mathbf{H}^{0}=\left(0, H_{0}, 0\right)$ and weakened by cylindrical cavities along the $z$-axis.

Under mechanical action that deforms the elastic medium an additional electromagnetic field is induced in the body and can be considered quasistatic $(\mathbf{D}=\mathbf{0}, \partial \mathbf{D} / \partial t=\mathbf{0}$, where $\mathbf{D}$ is the displacement current, and $t$ is time). Maxwell's equations and the equations of motion, after linearization $[3,5]$, will be the following:

$$
\begin{gathered}
\mathbf{h}=\operatorname{curl}\left(\mathbf{U} \times \mathbf{H}^{0}\right), \quad \mathbf{e}=-\mu_{\varepsilon}\left(\frac{\partial \mathbf{U}}{\partial t} \times \mathbf{H}^{0}\right), \\
\mu \nabla^{2} \mathbf{U}+(\lambda+\mu) \text { grad div } \mathbf{U}+\mu_{e} \operatorname{curl} \mathbf{h} \times \mathbf{H}^{0}=\rho \frac{\partial^{2} \mathbf{U}}{\partial t^{2}},
\end{gathered}
$$

where $h$ and $\mathbf{e}$ are the intensities of the induced magnetic and electric fields, $\mathbf{U}$ is the elastic displacement vector, $\lambda$ and $\mu$ are the Lamé parameters, $\mu_{e}$ is the magnetic permeability, $\rho$ is the density of the medium, and $\nabla^{2}$ is the Laplacian.

In the case of steady-state deformation of a longitudinal shear $\left(\mathbf{U}=(0,0, w), w=\operatorname{Re}\left\{W(x, y) e^{-i \omega t}\right\}\right.$, and $\omega$ is the cyclic frequency) the system of equations (1) leads to the following differential equation with respect to the amplitude of the displacement $W(x, y)\left(\gamma_{2}\right.$ is the wave number):

$$
\nabla^{2} W+\chi^{2} \partial^{2} W / \partial y^{2}+\gamma_{2}^{2} W=0 \quad\left(\chi^{2}=\mu_{e} H_{0}^{2} / \mu, \gamma_{2}^{2}=\rho \omega^{2} / \mu\right) .
$$

The total stresses $\sigma_{x z}, \sigma_{y z}$ are composed of the mechanical stresses $\tau_{x z}, \tau_{y z}$ and the Maxwell stresses $t_{x z}, t_{y z}$, and can be expressed in terms of the displacement $w(x, y)$ using the formulas

$$
\sigma_{x z}=\tau_{x z}+t_{x z}, \quad \sigma_{y z}=\tau_{x z}+t_{y z}, \quad \tau_{x z}=\mu \partial w / \partial x, \quad \tau_{y z}=\mu \partial w / \partial y, \quad t_{x z}=0, \quad t_{y z}=\mu \chi^{2} \partial w / \partial y .
$$

We assume that the boundary planes of the layer are free of load

$$
\partial W / \partial x=0 \quad(x=0, x=a),
$$

that a magnetoelastic shear wave

$$
w_{0}=\operatorname{Re}\left\{W_{0} e^{-i \omega t}\right\}, \quad W_{0}=\tau \exp \left\{i \gamma_{2} y / \sqrt{1+\chi^{2}}\right\}
$$

Translated from Matematicheskie Metody i Fiziko-Mekhanicheskie Polya, No. 37, 1994, pp. 74-78. Original article submitted September 14, 1990. 
is propagating along the $y$-axis, and that a time-harmonic mechanical load $X_{n}=Y_{n}=0, Z_{n}=\operatorname{Re}\left\{Z e^{-t+t}\right\}$ possibly acts on the surface of the cavity $S_{j}(j=\overline{1, m})$.

Let $L_{j}$ be the line of intersection of the surface $S_{j}$ with the $x y$-plane and $\mathbf{n}=(\cos \psi, \sin \psi)$ the outward unit normal to $L_{j}$. Assume that $Z$ and the curvature of the $\operatorname{arc} L_{j}$ are functions of class $H$ on $L_{j}$ [2]. The boundary condition on the edge of the cavity can be written as

$$
\left.(\partial W / \partial n)\right|_{L_{j}}=\frac{1}{\mu} Z
$$

We now introduce the coordinates

$$
x_{1}=x, \quad y_{1}=y / \sqrt{1+\chi^{2}}
$$

in which the resolvent equation (2) transforms into the Helmholtz equation

$$
\partial^{2} W / \partial x_{1}^{2}+\partial^{2} W / \partial y_{1}^{2}+\gamma_{2}^{2} W=0
$$

The differentials $d s_{1}$ and $d s$ of the arc $L_{j}$ in the $x_{1} y_{1}$ and $x y$ coordinates are connected by the relations

$$
d s_{1}=\sqrt{\left(1+\chi^{2} \sin ^{2} \psi\right) /\left(1+\chi^{2}\right)} d s
$$

We represent the solution of the boundary-value problem (2), (3)-(5) as the superposition $W=W_{0}+W_{1}$, where $W_{1}(x, y)$ is the scattering amplitude of the wave. We represent the latter as a single-layer potential [1]:

$$
W_{1}=\int_{L} p(s) G d s_{1} .
$$

Here $L_{j}=\bigcup_{j=1}^{\infty} L_{j}, p(s)$ is the unknown density, and $G$ is the Green's function for the boundary-value problem (6), (3) for the strip $0 \leq x_{1} \leq a,-\infty<y_{1}<\infty$ :

$$
\begin{gathered}
G=\frac{1}{2 i \gamma_{2} a} e^{i \gamma_{2}\left|\eta_{1}-y_{1}\right|}-\frac{1}{a} \sum_{k=1}^{\infty} \frac{1}{\lambda_{k}} e^{-\lambda_{k}\left|\eta_{1}-y_{1}\right|} \cos \alpha_{k} \xi \cos \alpha_{k} x, \\
\lambda_{k}=\sqrt{\alpha_{k}^{2}-\gamma_{2}^{2}} \quad\left(\gamma_{2}<\alpha_{k}\right), \quad \lambda_{k}=-i \sqrt{\gamma_{2}^{2}-\alpha_{k}^{2}} \quad\left(\gamma_{2}>\alpha_{k}\right), \quad \alpha_{k}=\frac{\pi k}{a}, \quad \eta_{1}=\eta / \sqrt{1+\chi^{2}} .
\end{gathered}
$$

The integral representation (7) satisfies the differential equation (2), the boundary conditions (3), and the radiative conditions of $[6]$.

In the case when $\eta_{1}=y_{1}$ the series in formula (8) converges conditionally, and for $\xi=x, \eta_{1}=y_{1}$ it becomes divergent. To get rid of this effect we single out its principal part, obtaining

$$
\begin{gathered}
G=G_{0}+G_{1}, \quad G_{0}=\left.\left(G-\frac{1}{2 i \gamma_{2} a}\right) e^{i \gamma_{2}\left|\eta_{1}-y_{1}\right|}\right|_{\gamma=0} \\
=\frac{1}{2 \pi} \ln \left|4 \sin \frac{\pi}{2 a}\left(\zeta_{1}-z_{1}\right) \sin \frac{\pi}{2 a}\left(\zeta_{1}+\bar{z}_{1}\right)\right|-\frac{1}{2 a}\left|\eta_{1}-y_{1}\right| \\
G_{1}=\frac{1}{2 i \gamma_{2} a} e^{i \gamma_{2}\left|\eta_{1}-y\right|}-\frac{1}{a} \sum_{k=1}^{\infty}\left(\frac{1}{\lambda_{k}} e^{-\lambda_{k}\left|\eta_{1}-y_{1}\right|}-\frac{1}{\alpha_{k}} e^{-\alpha_{k}\left|\eta_{1}-y_{1}\right|}\right) \cos \alpha_{k} \xi \cos \alpha_{k} x, \quad \zeta_{1}=\xi+i \eta_{1}, \quad z_{1}=x+i y_{1}
\end{gathered}
$$

It can be seen from this that the function $G$ of $(9)$ satisfies Eq. (2) with $\zeta \neq z$ and has a logarithmic singularity as $r=|z-\zeta| \rightarrow 0$. The general term of the series for $G_{1}$ dies out like $k^{-3}$ when $r=0$ and exponentially when $\eta \neq y$. The Green's function in the form (9) admits differentiation with respect to its variables. 
Computing the normal derivative of the function $W$ and substituting its limiting value as $z \rightarrow \zeta_{0}=$ $\xi_{0}+i \eta_{0} \in L$ into the boundary condition (5), we arrive at an integral equation with respect to the function $p(x)$ :

$$
\begin{gathered}
b\left(\psi_{0}\right) p\left(s_{0}\right)+\int_{L} p(s) M\left(s, s_{0}\right) d s=f\left(s_{0}\right) \\
b\left(\psi_{0}\right)=-\frac{1}{2}\left(1+\chi^{2} \sin ^{2} \psi_{0}\right)^{-\frac{1}{2}}, \quad \psi_{0}=\left.\psi\right|_{\zeta=\zeta_{0}}, \quad s_{0}=\left.s\right|_{\zeta=\zeta_{0}} \\
M\left(s, s_{0}\right)= \\
\left(2 a \sqrt{1+\chi^{2}} b(\psi)\right)^{-1}\left\{\frac{1}{4} \operatorname{Re}\left[\left(\cos \psi_{0}+\frac{i}{\sqrt{1+\chi^{2}}} \sin \psi_{0}\right)\left(\cot \frac{\pi}{2 a}\left(\zeta_{1}-\zeta_{01}\right)-\cot \frac{\pi}{2 a}\left(\bar{\zeta}_{1}+\zeta_{01}\right)+2 i \operatorname{sgn} \eta_{2}\right)\right]\right. \\
-\sum_{k=1}^{\infty}\left[\alpha_{k}\left(\frac{1}{\lambda_{k}} e^{-\lambda_{k}\left|\eta_{2}\right|}-\frac{1}{\alpha_{k}} e^{-\alpha_{k}\left|\eta_{2}\right|}\right) \sin \alpha_{k} \xi_{0} \cos \psi_{0}-\right. \\
\left.\left.\frac{1}{\sqrt{1+\chi^{2}}} \operatorname{sgn} \eta_{2}\left(e^{-\lambda_{k}\left|\eta_{2}\right|}-e^{-\alpha_{k}\left|\eta_{2}\right|}\right) \cos \alpha_{k} \xi_{0} \sin \psi_{0}\right] \cos \alpha_{k} \xi+\frac{1}{2 \sqrt{1+\chi^{2}}} \operatorname{sgn} \eta_{2} \cdot e^{i \gamma_{2}\left|\eta_{2}\right|} \sin \psi_{0}\right\} \\
f\left(s_{0}\right)=\frac{1}{\mu} Z-\frac{\tau}{2 a \sqrt{1+\chi^{2}}} e^{i \gamma_{2} \eta_{01}} \sin \psi_{0}, \quad \zeta_{01}=\xi_{0}+i \eta_{01}, \quad \eta_{01}=\eta_{0} / \sqrt{1+\chi^{2}}, \quad \eta_{2}=\eta_{1}-\eta_{01} .
\end{gathered}
$$

In Eq. (10) the kernel $M\left(s, s_{0}\right)$ has the estimate

$$
M\left(s, s_{0}\right)=-\left(\sqrt{1+\chi^{2}}-1\right) \sin \psi_{0} \sin \alpha_{1} \sqrt{1+\chi^{2} \sin ^{2} \psi_{0}} /\left(2 \pi r_{1}\left(1+\chi^{2}\right)\right)+O(1), \quad \zeta_{01}-\zeta_{0}=r_{1} e^{i \alpha_{1}}
$$

as $s \rightarrow s_{0}$. Consequently the integral equation (10) is singular for $\chi \neq 0$, while for $\chi=0$ it degenerates into a Fredholm equation. In both cases it has a unique solution in the class $H$ [2].

The integral equation (10) was solved numerically for the case of a single cavity using the method of mechanical quadratures [4]. In the process Eq. (10) was satisfied at the nodes $\theta_{i}=2 \pi(i-1) / n, i=1, \ldots, n$, and reduced to a system of linear algebraic equations with respect to the unknown quantities $p\left(s\left(\theta_{j}\right)\right)$, $\theta_{j}=\pi(2 j-1) / n, j=1, \ldots, n$, where the parametric representation of the edge $L$ was chosen in the form $\xi=\xi(\theta), \eta=\eta(\theta), 0 \leq \theta \leq 2 \pi$. The rectangular quadrature formula having its highest degree of algebraic precision in the case of periodic functions for both regular and singular integrals with the set of nodes $\theta_{i}$ and $\theta_{j}$ indicated above was applied to the integral in (10). The values of the density outside the integral at the nodes $\theta_{i}$ were determined using the interpolating polynomial of the function $p(s(\theta))$ over the nodes $\theta_{j}$ :

$$
p\left(s\left(\theta_{j}\right)\right)=\frac{1}{n} \sum_{j=1}^{n} p\left(s\left(\theta_{j}\right)\right)(-1)^{i+j} \csc \frac{\pi}{2 n}(2 i-2 j+1) .
$$

We carried out computations of the stresses $\sigma_{s z}=-\sigma_{x z} \psi+\sigma_{y z} \cos \psi, \sigma_{n z}=\sigma_{x z} \cos \psi+\sigma_{y z} \sin \psi=$ $t_{y z} \sin \psi$ on the surface of the cavity for the diffraction of an incident wave $(\tau \neq 0)$ on a cavity $(Z=0)$ free of force. The edge of the cavity was given in the form $\xi / a=b+c \cos \theta, \eta / a=d \sin \theta, 0 \leq \theta \leq 2 \pi$; for a circular cavity we took the values $b=0.5, c=d=0.2$, and for an elliptic cavity we took $b=0.5, c=0.2$, $d=0.1$.

Figures 1-4 show the distribution of stresses on the edge of a circular cavity (Figs. 1,2) and an elliptic cavity (Figs. 3, 4) for two values of the normalized wave number $\gamma_{2} a=\pi$ (Figs. 1, 3) and $11 \pi / 6$ (Figs. $2,4)$. The dimensionless quantities $\delta_{1}$ and $\delta_{2}$ are equal respectively to the ratios of the absolute values of the stress amplitudes $\sigma_{s z}$ and $\sigma_{n z}$ on the edge $L$ to the quantity $\left|T_{y}\right|=\mu \gamma_{2} \tau / \sqrt{1+\chi^{2}}$, where $T_{y}$ is the amplitude of the mechanical stress $\tau_{y z}$ in the incident wave. Curres 1-3 correspond to the variation of the quantity $\delta_{1}$, curves $4-5$ to the quantity $\delta_{2}$. Curve 1 is constructed for the value $\chi=0$ (no magnetic field), curves 2. 4 for $\chi=0.5$, and curves 3,5 for $\chi=1$. 


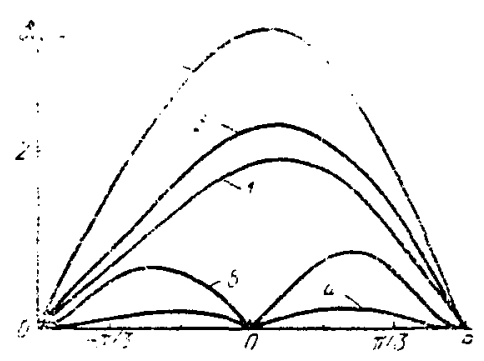

Fig. 1

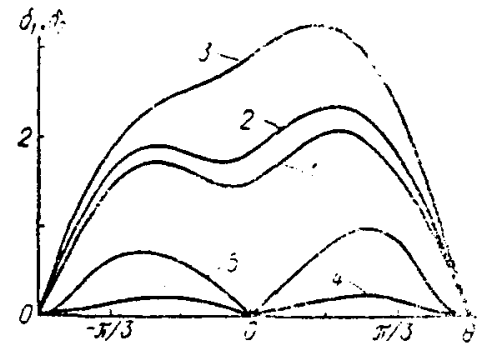

Fig. 2

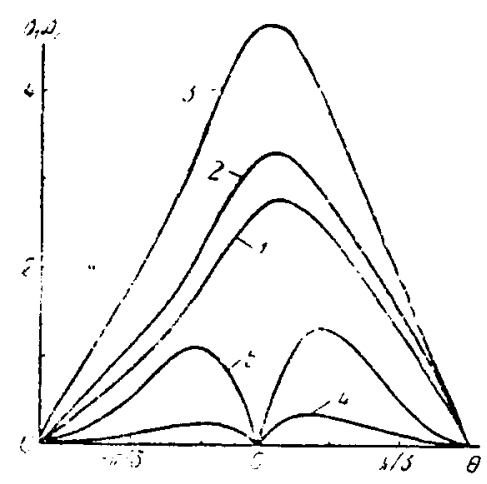

Fig. 3

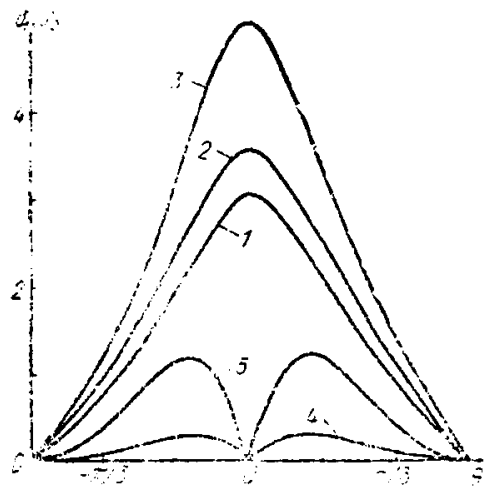

Fig. 4

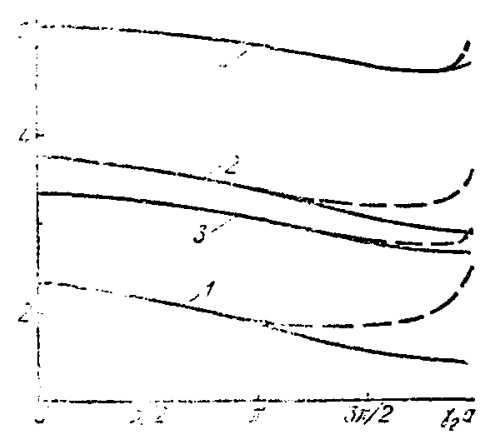

Fig. 5

Figure 5 shows the variation of the quantity $\delta_{1}$ for a circular cavity $(\chi=0$ in curve $1, \chi=1$ in curve 2) and an elliptic cavity ( $\chi=0$ in curve $3, \chi=1$ in curve 4 ) as functions of the parameter $\gamma_{2} a$. The solid lines correspond to the stress $\sigma_{s z}$ at the point $\theta=0$, the dashed lines to the Maxwell stress $\sigma_{s z}$.

The functions presented show that the maximal stress $\sigma_{s z}$ for $\gamma_{2} a<\pi$ is observed near the point $\theta=0$. With approach to the sliding point $\gamma_{2} a=2 \pi$ a significant redistribution of stresses occurs, and the maximal value of $\sigma_{s z}$ shifts to the umbral zone of the incident wave $(\theta>0)$. Here if the value of $\sigma_{s z}$ at $\theta=0$ passes uniformly through the sliding point (in the case of a circular cavity), then for the maximal stress on the edge the sliding point is a resonance point.

The application of a static magnetic field significantly increases the stresses. Thus in the examples under consideration with $\chi=1$ the quantity $\max _{\theta} \delta_{1}$ increases by $50-80 \%$, corresponding to a $30-60 \%$ increment in the maximal stress $\sigma_{s z}$ on $L$. Along with this the magnetic field partly counterbalances the dynamic effects. To be specific, it equalizes the stresses along the edge somewhat and prevents the shift of the maximum of $\sigma_{s z}$ away from the value $\theta=0$ as the sliding point is approached. In addition the magnetic field generates a stress $t_{n z}$ that satisfies $t_{n z}=0$ when $\theta=0$ and reaches its maximal value at $\theta \approx \pi / 4$ and $\pi / 6$ respectively for a circular cavity and an elliptic cavity.

\section{Literature Cited}

1. V. M. Babich, M. B. Kapilevich, S. G. Mikhlin, et al., The Linear Equations of Mathematical Physics [in Russian], Nauka, Moscow (1964).

2. N. I. Muskhelishvili, Singular Integral Equations [in Russian], Fizmatgiz, Moscow (1962).

3. W. Nowacki, Electromagnetic Effects in Solid Bodies [Russian translation], Mir, Moscow (1986). 
4. V. V. Panasyuk, M. P. Savruk, and Z. T. Nazarchuk, The Method of Singular Integral Equations in Two-Dimensional Diffraction Problems [in Russian], Naukova Dumka, Kiev (1984).

5. Ya. S. Podstrigach, Ya. I. Burak, A. P. Gachkevich, and L. V. Chernyavskaya. Thermoelasticity of Electrically Conducting Bodies [in Russian], Naukova Dumka, Kiev (1977).

6. A. G. Sveshnikov, "The principle of limiting absorption for a waveguide," Dokl. Akad. Nauk SSSR, 80, No. 3, 345-347 (1951).

7. Y. Shindo, "Diffraction of transient horizontal shear waves by a finite crack in the presence of a magnetic field," Lett. Appl. Eng. Sci., 3, No,. 5, 313-329 (1975).

8. Y. Shindo, "Diffraction of antiplane shear waves by a finite crack in the presence of a magnetic field," Z. angew. Math. Mech., 56, No. 1, 33-41 (1976).

9. Y. Shindo, "Diffraction of normal compression waves by a penny-shaped crack in the presence of an axial magnetic field," Int. J. Eng. Sci., 17, No. 5, 651-658 (1979). 